\title{
Effect of mouse antisera targeting the Phlebotomus papatasi midgut chitinase PpChit1 on sandfly physiology and fitness
}

\author{
Maricela Robles-Murguia', Nicholas Bloedow ${ }^{2}$, Leigh Murray², Marcelo Ramalho-Ortigão1/+ \\ ${ }^{1}$ Department of Entomology ${ }^{2}$ Department of Statistics, Kansas State University, Manhattan, KS, USA
}

\begin{abstract}
In sandflies, the absence of the peritrophic matrix (PM) affects the rate of blood digestion. Also, the kinetics of $P M$ secretion varies according to species. We previously characterised PpChit1, a midgut-specific chitinase secreted in Phlebotomus papatasi (PPIS) that is involved in the maturation of the PM and showed that antibodies against PpChit1 reduce the chitinolytic activity in the midgut of several sandfly species. Here, sandflies were fed on red blood cells reconstituted with naïve or anti-PpChit1 sera and assessed for fitness parameters that included blood digestion, oviposition onset, number of eggs laid, egg bouts, average number of eggs per bout and survival. In PPIS, anti-PpChit1 led to a one-day delay in the onset of egg laying, with flies surviving three days longer compared to the control group. Anti-PpChitl also had a negative effect on overall ability of flies to lay eggs, as several gravid females from all three species were unable to lay any eggs despite having lived longer than control flies. Whereas the longer survival might be associated with improved haeme scavenging ability by the PM, the inability of females to lay eggs is possibly linked to changes in PM permeability affecting nutrient absorption.
\end{abstract}

Key words: sandflies - PpChit1 - peritrophic matrix - blood digestion

Upon blood feeding, certain Diptera species such as mosquitoes and sandflies secrete a peritrophic matrix (PM) type 1 that completely surrounds the blood bolus. In addition to the compartmentalisation of the blood, the PM in these insects can also protect the midgut epithelia against abrasion and can function as a barrier to parasites or pathogens. The secretion of the PM type 1 by cells lining the midgut wall is stimulated by and occurs following the distention of the midgut as the insect blood feeds. Such is fundamentally distinct from PM type 2, which is constitutively secreted by specialised cells of the stomodeal valve (or cardia) in immature stages in mosquitoes and sandflies and in adults of insects such as tsetse fly.

In sandflies, it has been shown that the kinetics of PM secretion and its maturation can change according to species (Pimenta et al. 1997, Sadlova \& Volf 2009). PM maturation and degradation are regulated by chitinases secreted in the midgut lumen also following a blood meal (Ramalho-Ortigão \& Traub-Cseko 2003, Ramalho-Ortigão et al. 2005). Indication that the PM is modulated by the action of chitinases was obtained by feeding allosamidin, a chitinase inhibitor, to sandflies leading to a thicker and more persistent PM (Pimenta et al. 1997). This more robust PM, likely a result of the accumulation of chitin without a corresponding modu-

\footnotetext{
doi: 10.1590/0074-0276140382

Financial support: NIH RO1 (AI074691) (to MR-O)

The results herein are part of the MSc dissertation of MR-M (contribution 15-147-J from the Kansas Agricultural Experiment Station).

+ Corresponding author: mortigao@ksu.edu

Received 15 October 2014

Accepted 27 November 2014
}

lation and/or degradation by chitinase also was associated with a slower rate of excretion of the blood meal in sandflies (Pimenta et al. 1997). Finally, disruption of the PM in sandflies by addition of exogenous chitinase was recently shown to cause a speedier digestion of the blood meal, with faster onset of egg laying, but with fewer eggs laid compared to control flies and greater mortality of flies (de Araújo et al. 2012)

With the characterisation of the sandfly midgut chitinases LlChit1 (Ramalho-Ortigão \& Traub-Cseko 2003) and PpChitl (Ramalho-Ortigão et al. 2005), followed by RNAi knockdown studies of PpChitl (Coutinho-Abreu et al. 2010) and of the peritrophin PpPerl (Coutinho-Abreu et al. 2013), we reasoned that changes in the PM permeability, caused either by silencing (RNAi) or blocking (antibodies) of PM-related molecules, may affect digestion and/or excretion of the blood meal process in sandflies and consequently impact the fitness. Accordingly, here, we explored the possibility that feeding antibodies specific to PpChitl will reduce (or prevent) the degradation of the sandfly PM, thus delaying digestion and/or excretion of the blood and likely affecting egg laying as well as other fitness parameters such as survival. Anti-PpChitl was shown to have a cross-species effect, not only inhibiting the midgut chitinolytic of Phlebotomus papatasi (PPIS), but also that of Phlebotomus argentipes and Phlebotomus duboscqi (PDMA) (Ramalho-Ortigão et al. 2005). Thus, anti-PpChit1 antiserum was fed to three different sandfly species, PPIS, PDMA and Lutzomyia longipalpis (LLJB) using an artificial blood feeding apparatus.

Although no clear difference in the rate of blood meal digestion was observed, as we predicted, at least for PPIS sandflies certain fitness parameters were affected by feeding red blood cells (RBCs) reconstituted in anti-PpChitl compared to flies fed on RBCs reconstituted with naïve sera. Taken together, results from this study combined with our previous data (Coutinho-Abreu 
et al. 2010) suggest that PpChit1 and their respective orthologs are involved in the maturation of the sandfly PM. Targeting PpChitl will help us understand several aspects of the biology of sandfly vectors and provide further insights towards the development of novel strategies to control transmission of Leishmania by sandflies.

\section{MATERIALS AND METHODS}

Ethics statement - The use of animals during this study was reviewed and approved by the Kansas State University (KSU) Institutional Animal Care and Use Committee, United Sates of America.

Anti-PpChit1 sera - Antisera specific to PpChit1 were obtained as described previously (Coutinho-Abreu et al. 2010). Briefly, plasmid containing the mature PpChit1 cDNA was purified according to Ramalho-Ortigão et al. (2005). Prior to injection, samples were filter sterilised through a $0.2 \mu \mathrm{M}$ filter unit (Millipore). Purified plasmid was injected subcutaneously into the ears of isofluraneanesthetised female BALB/c mice, eight-12 weeks of age, using a 29.5 -gauge needle $(10 \mu \mathrm{g}$ of plasmid per mice ear). All mice were bred and maintained under pathogenfree conditions at the Division of Veterinary Medicine, KSU. Two weeks after the last DNA immunisation, approximately $300 \mu \mathrm{L}$ of blood was collected from the submandibular vein ("cheek bleed") of immunised animals. Sera were separated following centrifugation at 7,500 rpm for 10 min (Eppendorf, USA). Antibody titre was obtained for each mouse injected with the plasmid carrying the mature $P p C h i t 1$ (Supplementary data, Table I). Titres were measured with the Easy-Titer IgG Assay Kit (Pierce, USA) following manufacturer's instructions and kept at $-20^{\circ} \mathrm{C}$ until use. Serum obtained from mouse \#4 (Supplementary data, Table I) was used to feed the flies.

Sandfly rearing and blood feeding - PPIS Israeli strain, PDMA Mali strain and LLJB strain were reared in the Department of Entomology, KSU, as described (Coutinho-Abreu et al. 2010). Prior to blood feeding, sandflies of each species were separated into two 150 females and 30 males groups each containing four-to-five day old sandflies and placed in a $500 \mathrm{~mL}$ plastic container (Thermo-
Nalgene, USA) covered with a piece of nylon mesh (0.5 $\mathrm{mm})$. For blood feeding, RBCs collected from a naïve mouse were separately reconstituted in $195 \mu \mathrm{L}$ of naïve or in anti-PpChit1 sera (113 $\mu \mathrm{g}$ of total IgG) (Supplementary data, Table I). Assuming an IgG concentration of 0.5 $\mu \mathrm{g} / \mu \mathrm{L}$ for anti-PpChit1, a fully fed fly $(0.5 \mu \mathrm{L}$ of blood $)$ took in roughly $0.25 \mu \mathrm{g}$ of specific anti-PpChitl serum. Sandfly feeding was performed using a glass feeding apparatus fitted with pig intestine membrane.

Flies were allowed to feed for $1 \mathrm{~h}$. Immediately after feeding, fully engorged females were separated from the partial and non-fed ones by anesthetising them with $\mathrm{CO}_{2}$ and observing the midgut distension under a stereomicroscope (Carl Zeiss, USA). Only fully fed sandflies of similar size were used for all experiments. Sandflies were transferred to $250 \mathrm{~mL}$ paper cups (Huhtamaki, USA) and maintained at $26^{\circ} \mathrm{C}$ and $70 \%$ humidity with $20 \%$ sugar solution.

Analysis of blood digestion - The rate of haemoglobin excretion was assessed with a colorimetric assay using Drabkin's solution. Drabkin's solution was prepared by adding $5 \mu \mathrm{L}$ of $30 \%$ Brij-35 solution (Sigma, USA) to $10 \mathrm{~mL}$ of Drabkin's Reagent (Sigma) and mixing gently by inverting the tube several times. Following feeding, sandflies in either naïve sera or anti-PpChitl sera-reconstituted RBCs (described above) were selected for dissection at $24,30,36,48$ and $72 \mathrm{~h}$ post blood meal (PBM). Midguts were transferred into $250 \mu \mathrm{L}$ of Drabkin's solution, homogenised with a hand-held homogeniser (Kimble Chase, USA) for approximately $30 \mathrm{~s}$, transferred to a 96-well microtitre plate and incubated for $15 \mathrm{~min}$ at $25^{\circ} \mathrm{C}$. Absorbance at $540 \mathrm{~nm}$ was recorded on a Biotek Epoch Gen5 (Biotek, USA). Known quantities of mouse blood were used as a standard and each sample was measured in triplicate. For further comparisons, pictures of the dissected midguts were taken using an AM423X Dino-Eye camera (Dinolite, Taiwan).

As a measure of blood meal loss over time, the haemoglobin concentration (of dissected midguts) was determined for each of the three species of sandflies included in the study. For each species, five replicates for PPIS and

\section{TABLE I}

Effect of anti-PpChitl on fitness parameters for Phlebotomus papatasi (PPIS), Phlebotomus duboscqi (PDMA) and Lutzomyia longipalpis (LLJB)

\begin{tabular}{|c|c|c|c|c|c|c|c|c|c|c|c|c|}
\hline Species & Group & $\begin{array}{c}\text { Sandflies } \\
\text { (n) }\end{array}$ & $\begin{array}{c}\text { Egg laying } \\
\text { onset } \\
\text { (days) }\end{array}$ & $\mathrm{p}$ & $\begin{array}{c}\text { Survival } \\
\text { (days) }\end{array}$ & $\mathrm{p}$ & $\begin{array}{c}\text { Eggs } \\
\text { (n) }\end{array}$ & $\mathrm{p}$ & $\begin{array}{l}\text { Egg } \\
\text { bouts }\end{array}$ & $\mathrm{p}$ & $\begin{array}{l}\text { Average eggs } \\
\text { per bout } \\
\text { (n) }\end{array}$ & $\mathrm{p}$ \\
\hline \multirow[t]{2}{*}{ PPIS } & Control & 53 & 6.40 & 0.074 & 7.73 & 0.078 & 45 & 0.158 & 1 & 0.378 & 37 & 0.312 \\
\hline & anti-PpChit1 & 53 & 7.49 & & 9.60 & & 55 & & 1 & & 43 & \\
\hline \multirow[t]{2}{*}{ PDMA } & Control & 44 & 9.27 & 0.560 & 9.44 & 0.561 & 28 & 0.227 & 1 & 0.948 & 25 & 0.249 \\
\hline & anti-PpChit1 & 47 & 8.14 & & 8.42 & & 36 & & 1 & & 31 & \\
\hline \multirow[t]{2}{*}{ LLJB } & Control & 32 & 5.54 & 0.469 & 7.01 & 0.8052 & 32 & 0.999 & 2 & 0.513 & 19 & 0.513 \\
\hline & anti-PpChitl & 36 & 5.27 & & 6.9 & & 32 & & 2 & & 16 & \\
\hline
\end{tabular}

fitness parameters were calculated by combining four, five and three experimental replicates for each species, respectively. 
PDMA and three replicates for LLJB were performed. Replicates were pooled together in the statistical analysis.

Sandfly oviposition and fitness - Three days PBM, fully fed females were individually transferred to $125-\mathrm{mL}$ plastic pots containing a thin layer (roughly $0.5 \mathrm{~cm}$ ) of plaster at the bottom (ovipot), so that one female was kept in each pot. All ovipots were maintained at approximately $65-70 \%$ humidity and each sandfly had access to $20 \%$ sugar solution ad libitum on a piece of embedded cotton placed on top of the mesh of each pot and replaced daily. The onset of oviposition (effective day on which eggs began to be laid), the number of eggs laid, the number of egg bouts, average number of eggs per bout and the survival of sandflies were recorded daily for each sandfly/ovipot for the duration of the experiment, until all flies died.

In this case, four replicates for PPIS, three replicates for PDMA and LLJB were completed. As before, replicates were pooled together in the statistical analysis.

Statistical analyses - To test the effect of anti-PpChit1, statistical analyses were conducted both by individual species and to compare species. The general experimental design for an individual species was a randomised complete block design (RCBD) with subsampling. The anti-PpChit1 was the treatment factor, the replicate was the blocking factor and individual flies were the subsamples. To compare species, a RCBD also was used, with a 2 (anti-PpChit1) x 3 (species) factorial as the treatment structure, the replicate nested within species as the blocking factor and individual flies as subsamples. Careful consideration was taken in selecting the type of probability distribution to be used with each response variable analysed as indicated below. Due to an unequal number of replications and subsamples per species, the between-within denominator degrees of freedom method (Schluchter \& Elashoff 1990) was used. In addition, to help evaluate the antibody treatment by species interaction, simple effects of antibody treatment vs. control were done for all species. Statistical analyses were conducted using the MIXED and GLIMMIX procedures, which implement the aforementioned betweenwithin method in SAS/STAT ${ }^{\circledR}$ software v.9.3 (SAS Institute Inc, USA). For technical details on SAS procedures used, see SAS Online Documentation (support.sas.com/ documentation/onlinedoc/stat/). A significance level of $\alpha=0.05$ was used for all tests, but results with $\alpha=0.10$ are discussed because the number of replications per species is small and these experiments may hence be thought of as "pilot" studies.

Analysis for blood digestion response - To investigate changes in haemoglobin concentration over specified time intervals, a split plot in time was added to the general experimental design described above. Differences in blood digestion between the control and the treatment groups were initially accessed via the MIXED procedure, allowing us to get variance estimates and account for the replicate-to-replicate variability. Since the overall residuals appeared rightwardly skewed and tests of normality from SAS ${ }^{\circledR}$ UNIVARIATE procedure (SAS/STAT ${ }^{\circledR}$ software v.9.3) showed residuals to be not normal, a generalised linear mixed model with a gamma distribution and a log link function in the SAS GLIMMIX procedure was used (Stroup 2012).

Analysis for oviposition and fitness responses - Due to the discrete nature of the measurements for onset of oviposition, survival and egg bouts, the SAS GLIMMIX procedure was used to fit a generalised linear mixed model with a Poisson distribution and a log link function to analyse these responses. In the case of the total number of eggs laid and average number of eggs per bout, a generalised linear mixed model with a gamma distribution and a $\log$ link function were fitted, again using the SAS GLIMMIX procedure to account for upper skew of the data.

Gravid flies that never laid eggs - A significant number of gravid flies never laid any eggs. To investigate differences in the proportion of gravid sandflies that never laid eggs in the control and the anti-PpChitl groups for an individual species, a Fisher's Exact Test was conducted using Minitab ${ }^{\circledR}$ Statistical software v.16 (Minitab Inc, USA).

\section{RESULTS}

Effect of anti-PpChit1 on blood retention - Overall, there was no statistical significant difference in blood retention in midguts from naïve blood-fed flies compared with anti-PpChit1 blood-fed flies when examined over time (Supplementary data, Figure, Tables I, II).

Interestingly, we observed a faster excretion of the blood meal in anti-PpChitl fed PDMA flies at the beginning with a decrease later during digestion. At $24 \mathrm{~h} \mathrm{PBM}$, the blood retained in the midgut of anti-PpChitl fed flies was equivalent to the control group. Then, at 30 and $36 \mathrm{~h}$ PBM anti-PpChitl fed flies begun to excrete the blood meal faster than the control group. At $48 \mathrm{~h}$ they seemed to slow down the digestion process compared to the control group. However, these results are not statistically significant.

Sandfly oviposition and fitness - A delay of approximately one day on the onset of oviposition was detected for PPIS flies (from 6.4 days for the onset in control flies to 7.5 days for PpChitl fed flies; $p=0.074$ ) (Table I). A difference in survival of PPIS flies, from 7.73 days for the control group to 10.6 days for anti-PpChit1 $(\mathrm{p}=0.078)$, also was observed. No significant difference was detected for the other fitness parameters. The analysis for PDMA and LLJB species did not show a statistically significant difference in any of the fitness parameters between the control and the anti-PpChitl groups (Table I).

Gravid sandflies that never laid eggs - During the study, a percentage of females from all three species of sandflies did not lay any eggs even though these were clearly gravid flies. For both PDMA and LLJB, more flies fed with anti-PpChit1 did not lay any eggs compared to the control groups (Table II). Fisher's exact test suggested a difference approaching significance for both PDMA ( $p$ $=0.058)$ and LLJB $(p=0.06)$ regarding this potentially negative effect of anti-PpChitl on egg laying. Interestingly, for the LLJB flies, nearly $20 \%$ of flies that fed on anti-PpChit1 did not lay eggs. For PDMA, this number was nearly $15 \%$. No significant difference $(p=0.319)$ was observed for PPIS. Curiously, we also noted that, for a 
TABLE II

Gravid sandflies that never laid eggs

\begin{tabular}{lcccccc}
\hline $\begin{array}{l}\text { Sandfly } \\
\text { species }\end{array}$ & Group & $\begin{array}{c}\text { Sandflies } \\
(\mathrm{n})\end{array}$ & $\begin{array}{c}\text { Flies that never laid eggs } \\
\mathrm{n}(\%)\end{array}$ & $\mathrm{p}$ & $\begin{array}{c}\text { Mean survival (SD)/ } \\
\text { never laid eggs }\end{array}$ & $\begin{array}{c}\text { Mean survival (SD)/ } \\
\text { laid eggs }\end{array}$ \\
\hline PPIS & Control & 53 & $7(13.2)$ & 0.319 & $15.14(4.14)$ & $7.49(1.23)$ \\
& anti-PpChit1 & 53 & $3(5.66)$ & & $15.66(4.72)$ & $9.6(1.7)$ \\
PDMA & Control & 44 & $1(2.27)$ & 0.058 & $10(-)$ & $9.27(2.34)$ \\
& anti-PpChit1 & 47 & $7(14.89)$ & & $12.71(5.53)$ & $8.14(1.9)$ \\
LLJB & Control & 32 & $1(3.13)$ & 0.06 & $10(-)$ & $7.0117(0.86)$ \\
& anti-PpChit1 & 36 & $7(19.44)$ & & $11.1(3.39)$ & $6.9(0.82)$ \\
\hline
\end{tabular}

total number of gravid flies per species and number of gravid flies per treatment that did not lay eggs are shown. The percentage of gravid flies per treatment group that did not lay eggs is shown and the p-values are given from the Fisher's Exact Test. The mean survival (in days) with the standard deviation (SD) is shown for gravid flies that never laid eggs vs. gravid flies that laid eggs. LLJB: Lutzomyia longipalpis; PDMA: Phlebotomus duboscqi; PPIS: Phlebotomus papatasi.

small sample size, PDMA and PPIS that were fed with anti-PpChitl had more eggs (81 and 72 eggs, respectively) in their ovaries than control flies (33 and 62 eggs); yet, these flies never laid eggs (Supplementary data, Table II). Due to the experimental design used in our studies and due to how the data were collected, we were unable to (statistically) compare the survival of the flies that successfully lay with flies that did not lay any eggs even though their survival was considerable. Nevertheless, the average number of days that flies lived under the experimental conditions described is shown in Table II.

Comparing effect of anti-PpChit1 across sandfly species - The effect of anti-PpChitl was also evaluated to compare the three species of sandflies for the blood digestion and all the fitness parameters mentioned above. No statistically significant differences were observed.

\section{DISCUSSION}

PpChitl is a midgut-specific chitinase presumably involved in modulation and degradation of the PM in PPIS and escape of parasites from the endoperitrophic space (Ramalho-Ortigão et al. 2005). It has been demonstrated that anti-PpChitl sera inhibit chitinolytic activity in the midgut of PPIS. Interestingly, these same antibodies displayed a cross-species effect, inhibiting chitinolytic activity in the midgut of PDMA and P. argentipes (Ramalho-Ortigão et al. 2005). We also showed that RNAi-induced knockdown of PpChitl leads to a significant reduction of Leishmania major within PPIS midgut (Coutinho-Abreu et al. 2010), which is associated with the thickening of the PM (unpublished observations). Thus, blocking PpChitl by means of feeding flies with polyclonal antisera specifically raised against this protein possibly changes the permeability of the PM in sandflies which in turn impacts fitness.

Anti-PpChit1 specific antiserum was fed to three different sandfly species, PPIS, PDMA and to LLJB using an artificial blood feeding apparatus. We then assessed the rate of blood digestion, or excretion, as well as fitness parameters such as the onset of oviposition, the total number of egg laid, eggs bouts, number of eggs per bout and sandfly overall survival.

Regarding the rate of excretion of the blood, as measured by the release of haemoglobin, no statistical significant differences were observed between anti-PpChitl fed or naïve sera fed flies for all three sandfly species tested (PPIS, PDMA and LLJB). These results are similar to observations by Pimenta et al. (1997) in which no difference in blood meal loss/excretion was observed when comparing between blood-fed flies, infected blood-fed flies and bloodfed treated with chitinase. However, Pimenta et al. (1997) did observe differences in blood meal loss in chitinasetreated infected flies compared to the other groups. Moreover, greatest decline of haemoglobin occurred between 48$72 \mathrm{~h}$ with complete loss of the blood meal by $96 \mathrm{~h}$.

An apparent difference was detected in the excretion of the blood in PDMA flies at 30 and $36 \mathrm{~h}$ PBM (Table III), as indicated by the concentration of haemoglobin in comparison to PPIS and LLJB (Tables IV, V). In this case, in PDMA fed on anti-PpChitl sera displayed a rate of digestion that was faster than naïve sera fed flies, whereas the opposite was observed in PPIS and LLJB for the same time points. Though no statistical difference was observed in any of the treatments in all three flies, it is plausible that in PDMA the PM kinetics affected the results observed. Another possibility is that upon feeding, chitinase is secreted into the gut lumen as an inactive pro-enzyme that is later activated by trypsin (Shen \& Jacobs-Lorena 1997). Thus, if inhibitors of trypsin are present in the gut between 24-48 h PBM, the activation of chitinase might not be achieved until the levels of this inhibitors decrease.

Haematophagy provides females with essential nutrients used for egg development (Villalon et al. 2003, Secundino et al. 2005). A slower blood digestion, to some extent expected after feeding with anti-PpChit1, would then lead to slower egg production (oviposition) due to longer lasting PM affecting absorption of nutrients. In our results, however, no difference in blood digestion between control and experimental flies of the three species (PPIS, PDMA and LLJB) was observed. 
TABLE III

Amount of blood in control and anti-PpChitl groups for Phlebotomus duboscqi (PDMA)

\begin{tabular}{|c|c|c|c|c|c|c|c|}
\hline \multirow[b]{2}{*}{$\begin{array}{l}\text { Time } \\
\text { PBM } \\
\text { (h) }\end{array}$} & \multicolumn{3}{|c|}{ Control } & \multicolumn{3}{|c|}{ anti-PpChit1 } & \multirow[b]{2}{*}{$\mathrm{p}$} \\
\hline & $\begin{array}{l}\text { Sandfly } \\
\text { PDMA } \\
\text { (n) }\end{array}$ & $\begin{array}{l}\text { Haemoglobin } \\
(\mathrm{mg} / \mathrm{mL})\end{array}$ & $95 \% \mathrm{CI}$ & $\begin{array}{c}\text { Sandfly } \\
\text { PDMA } \\
\text { (n) }\end{array}$ & $\begin{array}{l}\text { Haemoglobin } \\
(\mathrm{mg} / \mathrm{mL})\end{array}$ & $95 \% \mathrm{CI}$ & \\
\hline 24 & 25 & 0.261 & $0.216-0.315$ & 25 & 0.261 & $0.216-0.316$ & 0.987 \\
\hline 30 & 25 & 0.266 & $0.220-0.322$ & 25 & 0.244 & $0.202-0.295$ & 0.364 \\
\hline 36 & 26 & 0.247 & $0.204-0.298$ & 26 & 0.215 & $0.178-0.260$ & 0.142 \\
\hline 48 & 26 & 0.198 & $0.164-0.239$ & 26 & 0.222 & $0.183-0.268$ & 0.225 \\
\hline
\end{tabular}

five replicates performed and pooled together to generate the average haemoglobin concentration in whole midgut at different time points post blood meal (PBM). CI: confidence interval.

TABLE IV

Amount of blood in control and anti-PpChitl groups for Phlebotomus papatasi (PPIS)

\begin{tabular}{|c|c|c|c|c|c|c|c|}
\hline \multirow[b]{2}{*}{$\begin{array}{l}\text { Time PBM } \\
\text { (h) }\end{array}$} & \multicolumn{3}{|c|}{ Control } & \multicolumn{3}{|c|}{ anti-PpChit1 } & \multirow[b]{2}{*}{$\mathrm{p}$} \\
\hline & $\begin{array}{l}\text { Sandfly PPIS } \\
\text { (n) }\end{array}$ & $\begin{array}{l}\text { Haemoglobin } \\
\qquad(\mathrm{mg} / \mathrm{mL})\end{array}$ & $95 \% \mathrm{CI}$ & $\begin{array}{l}\text { Sandfly PPIS } \\
\text { (n) }\end{array}$ & $\begin{array}{l}\text { Haemoglobin } \\
\quad(\mathrm{mg} / \mathrm{mL})\end{array}$ & $95 \% \mathrm{CI}$ & \\
\hline 30 & 30 & 0.268 & $0.200-0.359$ & 32 & 0.262 & $0.196-0.351$ & 0.863 \\
\hline 36 & 30 & 0.258 & $0.193-0.346$ & 32 & 0.282 & $0.211-0.378$ & 0.497 \\
\hline 48 & 36 & 0.221 & $0.165-0.295$ & 36 & 0.221 & $0.165-0.296$ & 0.982 \\
\hline 72 & 32 & 0.132 & $0.098-0.177$ & 52 & 0.15 & $0.112-0.200$ & 0.326 \\
\hline
\end{tabular}

five replicates were measured and pooled together to generate the average of haemoglobin concentration in whole midgut at different time points post blood meal (PBM). CI: confidence interval.

Regarding the number of eggs laid by females fed either with anti-PpChitl or naïve sera, no difference was observed for any of the three sandfly species, PPIS, PDMA or LLJB. In a similar fashion, no difference in reproduction and fecundity (egg production) were observed in Aedes stephensi and Aedes aegypti fed with antisera targeting the PM (Villalon et al. 2003, Kato et al. 2008).

Interestingly, a difference that can be considered as "approaching significance" with regards to the onset of oviposition in PPIS flies fed with anti-PpChit1 was observed. PPIS fed on anti-PpChit1 sera took approximately one extra day to start laying eggs in comparison to the flies that were fed the naiive sera. Recently, it has been shown that oviposition onset of LLJB fed on blood containing exogenous chitinase occurred sooner and produced fewer eggs than control fed flies (de Araújo et al. 2012). Taken together these data suggest that, following digestion of the blood meal, the presence of the PM in sandflies somehow slows down the absorption of nutrients that are used for egg development and egg laying.

Regarding survival, a slight difference "approaching significance" $(p=0.078)$ was observed in PPIS flies that fed on blood containing anti-PpChitl in comparison to flies that fed on naïve sera: PPIS flies fed on antiPpChit1 lived approximately three days longer. It is conceivable that, at least for PPIS, the feeding of antiPpChitl leading to a thicker PM possibly provides additional haeme binding sites (haeme-regulatory motifs from peritrophins associated with the PM) to further reduce the toxic effects of this molecule.

Gravid PDMA and LLJB sandflies treated with antiPpChit1 did not lay eggs (p-values at 0.058 and 0.06 , respectively, and deemed "approaching significance") even when surviving longer than flies that laid eggs. For LLJB, nearly $20 \%$ of flies fed on anti-PpChit1 did not lay their eggs compared to approximately $3 \%$ of flies that fed the control sera. For PDMA, this difference was roughly $15-2.3 \%$. To our surprise, this effect was not reproduced in the PPIS flies. It is not yet clear if the difference observed regarding the ability to lay eggs was due, for example, to variability related to the size of the blood meal, the body size, the sugar diet or whether these females had mated successfully or whether this represents a possible effect of the anti-PpChitl sera. Regarding the first three variables (blood meal size, sandfly body size and sugar diet), our experimental design was done to 
TABLE V

Amount of blood in control and anti-PpChitl groups for Lutzomyia longipalpis (LLJB)

\begin{tabular}{|c|c|c|c|c|c|c|c|}
\hline \multirow[b]{2}{*}{$\begin{array}{l}\text { Time PBM } \\
\text { (h) }\end{array}$} & \multicolumn{3}{|c|}{ Control } & \multicolumn{3}{|c|}{ anti-PpChit1 } & \multirow[b]{2}{*}{$\mathrm{p}$} \\
\hline & $\begin{array}{l}\text { Sandfly LLJB } \\
\text { (n) }\end{array}$ & $\begin{array}{l}\text { Haemoglobin } \\
\qquad(\mathrm{mg} / \mathrm{mL})\end{array}$ & $95 \% \mathrm{CI}$ & $\begin{array}{l}\text { Sandfly LLJB } \\
\text { (n) }\end{array}$ & $\begin{array}{l}\text { Haemoglobin } \\
\qquad(\mathrm{mg} / \mathrm{mL})\end{array}$ & $95 \% \mathrm{CI}$ & \\
\hline 24 & 20 & 0.265 & $0.189-0.371$ & 22 & 0.283 & $0.202-0.396$ & 0.620 \\
\hline 30 & 20 & 0.272 & $0.194-0.381$ & 20 & 0.307 & $0.219-0.43$ & 0.368 \\
\hline 36 & 20 & 0.252 & $0.180-0.354$ & 22 & 0.304 & $0.217-0.425$ & 0.179 \\
\hline 48 & 22 & 0.261 & $0.186-0.366$ & 21 & 0.281 & $0.201-0.394$ & 0.577 \\
\hline
\end{tabular}

three replicates were performed and pooled together to generate the average of haemoglobin concentration in whole midguts at different time points post blood meal (PBM). CI: confidence interval.

minimise as much as possible the influence if these factors in our analyses. Regarding successful mating, it is known that male accessory gland secretions are able to influence various aspects of female reproductive physiology and behaviour, including oviposition (Gillot 2003, Avila et al. 2011). Notwithstanding, if an effect of antiPpChitl is indeed the cause for such an effect, the precise mechanism(s) remains to be investigated.

In sandflies, the PM was associated with the rate of excretion (Pimenta et al. 1997). Accordingly, absence of the PM due to the presence of exogenous chitinase in the blood meal was correlated with a speedier digestion and faster onset of egg laying. However, it also led to a greater number of flies dying without laying eggs and to fewer eggs been laid per female (de Araújo et al. 2012).

In spite of the subtle differences observed for most of the parameters investigated, the results from this study are consistent with data previously reported (de Araújo et al. 2012). Moreover, the presence of proteases in the midgut of the sandflies soon after blood feeding likely affected the bioavailability of the anti-PpChitl antibodies, masking or reducing their effect. The results presented here provide further evidence of the role played by PpChit1 in modulating the PM. However, how the permeability of the PM can influence nutrients absorption affecting longevity and oviposition in sandflies remains to be investigated.

\section{REFERENCES}

Avila FW, Sirot LK, La Flamme BA, Rubinstein CD, Wofner MF 2011. Insect seminal fluid proteins: identification and function. Annu Rev Entomol 56: 21-40.

Coutinho-Abreu IV, Sharma NK, Robles-Murguia M, RamalhoOrtigão M 2010. Targeting the midgut secreted PpChit1 reduces Leishmania major development in its natural vector, the sand fly Phlebotomus papatasi. PLoS Negl Trop Dis 4: e901.

Coutinho-Abreu IV, Sharma NK, Robles-Murguia M, Ramalho-Ortigão M 2013. Characterization of Phlebotomus papatasi peritrophins and the role of PpPerl in Leishmania major survival in its natural vector. PLoS Negl Trop Dis 7: e2132.

de Araújo APO, Telleria EL, Dutra JMF, Júlio RM, Traub-Cseko YM 2012. Disruption of the peritrophic matrix by exogenous chitinase feeding reduces fecundity in Lutzomyia longipalpis females. Mem Inst Oswaldo Cruz 107: 543-545.

Gillot C 2003. Male accessory gland secretions: modulators of female reproductive physiology and behavior. Annu Rev Entomol 48: 163-184.

Kato N, Mueller CR, Fuchs JF, McElroy K, Wessely V, Higgs S, Christensen BM 2008. Evaluation of the function of a type I peritrophic matrix as a physical barrier for midgut epithelium invasion by mosquito-borne pathogens in Aedes aegypti. Vector Borne Zoonotic Dis 8: 701-712.

Pimenta PF, Modi GB, Pereira ST, Shahabuddin M, Sacks DL 1997. A novel role for the peritrophic matrix in protecting Leishmania from the hydrolytic activities of the sand fly midgut. Parasitology 115: 359-369.

Ramalho-Ortigão JM, Kamhawi S, Joshi MB, Reynoso D, Lawyer PG, Dwyer DM, Sacks DL, Valenzuela JG 2005. Characterization of a blood activated chitinolytic system in the midgut of the sand fly vectors Lutzomyia longipalpis and Phlebotomus papatasi. Insect Mol Biol 14: 703-712.

Ramalho-Ortigão JM, Traub-Cseko YM 2003. Molecular characterization of Llchit1, a midgut chitinase cDNA from the leishmaniasis vector Lutzomyia longipalpis. Insect Biochem Mol Biol 33: 279-287.

Sadlova J, Volf P 2009. Peritrophic matrix of Phlebotomus duboscqi and its kinetics during Leishmania major development. Cell Tissue Res 337: 313-325.

Schluchter MD, Elashoff JD 1990. Small-sample adjustments to tests with unbalanced repeated measures assuming several covariance structures. J Stat Comput Simul 37: 69-87.

Secundino NFC, Nacif-Pimenta R, Hajmova M, Volf P, Pimenta PFP 2005. Midgut muscle network in Lutzomyia longipalpis and Phlebotomus duboscqi sand flies: spatial organization and structural modification after blood meal. Arthropod Struct Dev 34: 167-178.

Shen Z, Jacobs-Lorena M 1997. Characterization of a novel gut-specific chitinase gene from the human malaria vector Anopheles gambiae. J Biol Chem 272: 28895-28900.

Stroup WW 2012. Generalized linear mixed models: modern concepts, methods and applications, Chapman \& Hall/RC Press, Boca Raton, $555 \mathrm{pp}$.

Villalon JM, Ghosh A, Jacobs-Lorena M 2003. The peritrophic matrix limits the rate of digestion in adult Anopheles stephensi and Aedes aegypti mosquitoes. J Insect Physiol 49: 891-895. 

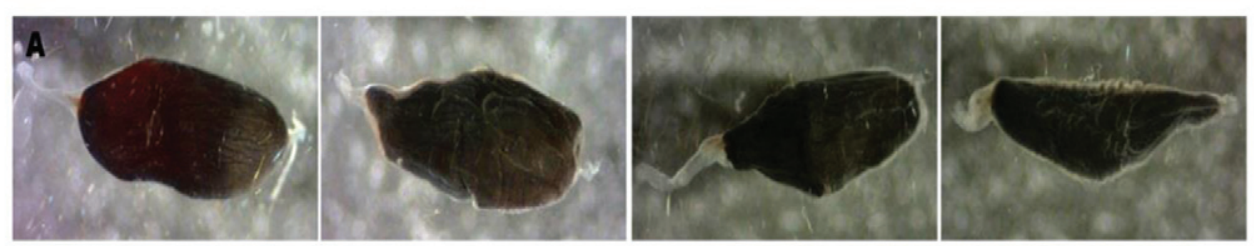

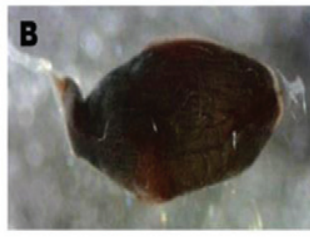

$24 \mathrm{~h}$

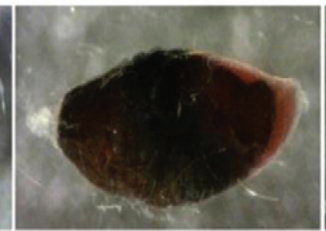

$30 \mathrm{~h}$

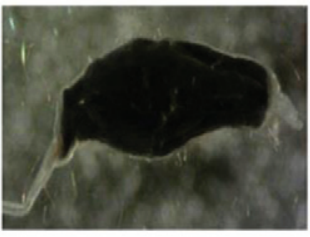

$36 \mathrm{~h}$

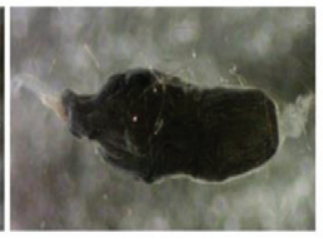

$48 \mathrm{~h}$

Sandfly midgut digestion process over time. Midguts of sandflies fed on red blood cells reconstituted in naïve (A) or anti-PpChit1 (B) sera and dissected at 24,30,36 and $48 \mathrm{~h}$ post blood ingestion. Midguts are displayed with their anterior end (or thoracic portion) to the left and the posterior end to the right.

TABLE I

Antibody titres of nine mice injected with plasmid

\begin{tabular}{|c|c|c|c|c|}
\hline Mouse & OD 340 & $\begin{array}{l}\text { Total IgG } \\
\text { concentration } \\
(\mathrm{mg} / \mathrm{mL})\end{array}$ & SD & $\begin{array}{l}\text { CV } \\
(\%)\end{array}$ \\
\hline \multirow[t]{2}{*}{$\# 1$} & 0.817 & 0.59 & 0.11 & 9.21 \\
\hline & 0.788 & & & \\
\hline \multirow[t]{2}{*}{$\# 2$} & 0.875 & 0.25 & 0.01 & 0.589 \\
\hline & 0.873 & & & \\
\hline \multirow[t]{2}{*}{$\# 3$} & 0.907 & 0.12 & 0.02 & 2.01 \\
\hline & 0.914 & & & \\
\hline \multirow[t]{2}{*}{$\# 4$} & 0.854 & 0.58 & 0.36 & 28.7 \\
\hline & 0.762 & & & \\
\hline \multirow[t]{2}{*}{$\# 5$} & 0.815 & 0.45 & 0.1 & 8.61 \\
\hline & 0.843 & & & \\
\hline \multirow[t]{2}{*}{ \#6 } & 0.85 & 0.32 & 0.04 & 3.89 \\
\hline & 0.863 & & & \\
\hline \multirow[t]{2}{*}{$\# 7$} & 0.928 & 0.06 & 0.02 & 2.26 \\
\hline & 0.936 & & & \\
\hline \multirow[t]{2}{*}{$\# 8$} & 0.867 & 0.28 & 0 & 0 \\
\hline & 0.867 & & & \\
\hline \multirow[t]{2}{*}{$\# 9$} & 0.835 & 0.37 & 0.06 & 6.05 \\
\hline & 0.855 & & & \\
\hline \multirow[t]{2}{*}{ Naïve mouse sera } & 0.943 & 0 & 0.04 & 6.43 \\
\hline & 0.966 & & & \\
\hline
\end{tabular}

the total concentration of IgG for each mouse was calculated based on absorbance at $340 \mathrm{~nm}$ of a 1:10.000 dilution. Optical density (OD) were plotted to determine each individual concentration. Each sample was done in duplicate. Standard deviation (SD) and coefficient of variance (CV) are shown for each pair of sample readings.
TABLE II

Number of eggs retained in ovaries

\begin{tabular}{lccc}
\hline Species & Group & $\begin{array}{c}\text { Sandflies } \\
\text { (n) }\end{array}$ & $\begin{array}{c}\text { Average } \\
\text { eggs in fly } \\
\text { (n) }\end{array}$ \\
\hline PPIS & Control & 7 & 62 \\
& anti-PpChit1 & 3 & 72 \\
PDMA & Control & 1 & 33 \\
\multirow{2}{*}{ LLJB } & anti-PpChit1 & 2 & 81 \\
\hline
\end{tabular}

after each sandfly died, its ovaries were dissected and the number of eggs left was counted. LLJB: Lutzomyia longipalpis; ND: not determined; PDMA: Phlebotomus duboscqi; PPIS: Phlebotomus papatasi. 